\title{
Primary Hypertrophic Osteoarthropathy
}

National Cancer Institute

\section{Source}

National Cancer Institute. Primary Hypertrophic Osteoarthropathy. NCI Thesaurus. Code C85023.

A rare disorder of unknown etiology characterized by hypertrophy of the bones of the distal extremities, periostosis of the tubular bones, digital clubbing, and skin changes including coarse facial features, acne, and hyperhydrosis. 